\title{
Antibody-independent isolation of circulating tumor cells by continuous-flow dielectrophoresis
}

\author{
Sangjo Shim, ${ }^{1,2}$ Katherine Stemke-Hale, ${ }^{3}$ Apostolia M. Tsimberidou, ${ }^{4}$ \\ Jamileh Noshari, ${ }^{1}$ Thomas E. Anderson, ${ }^{1}$ and Peter R. C. Gascoyne ${ }^{1, a)}$ \\ ${ }^{1}$ Department of Imaging Physics Research, The University of Texas, \\ M.D. Anderson Cancer Center, 1515 Holcombe Boulevard, Houston, Texas 77030, USA \\ ${ }^{2}$ Department of Biomedical Engineering, The University of Texas at Austin, \\ 1 University Station, C0800, Austin, Texas 78712, USA \\ ${ }^{3}$ Department of Systems Biology, The University of Texas, M.D. Anderson Cancer Center, \\ 1515 Holcombe Boulevard, Houston, Texas 77030, USA \\ ${ }^{4}$ Department of Investigational Therapeutics, The University of Texas, M.D. Anderson \\ Cancer Center, 1515 Holcombe Boulevard, Houston, Texas 77030, USA
}

(Received 27 August 2012; accepted 3 October 2012; published online 16 January 2013)

Circulating tumor cells (CTCs) are prognostic markers for the recurrence of cancer and may carry molecular information relevant to cancer diagnosis. Dielectrophoresis (DEP) has been proposed as a molecular marker-independent approach for isolating CTCs from blood and has been shown to be broadly applicable to different types of cancers. However, existing batch-mode microfluidic DEP methods have been unable to process $10 \mathrm{ml}$ clinical blood specimens rapidly enough. To achieve the required processing rates of $10^{6}$ nucleated cells $/ \mathrm{min}$, we describe a continuous flow microfluidic processing chamber into which the peripheral blood mononuclear cell fraction of a clinical specimen is slowly injected, deionized by diffusion, and then subjected to a balance of DEP, sedimentation and hydrodynamic lift forces. These forces cause tumor cells to be transported close to the floor of the chamber, while blood cells are carried about three cell diameters above them. The tumor cells are isolated by skimming them from the bottom of the chamber while the blood cells flow to waste. The principles, design, and modeling of the continuous-flow system are presented. To illustrate operation of the technology, we demonstrate the isolation of circulating colon tumor cells from clinical specimens and verify the tumor origin of these cells by molecular analysis. (C) 2013 American Institute of Physics.

[http://dx.doi.org/10.1063/1.4774304]

\section{INTRODUCTION}

Cancer cells shed from a primary tumor may enter the cardiovascular system as circulating tumor cells (CTCs). ${ }^{1}$ After invading tissues in distant organs, they may proliferate and form secondary tumors called metastases. CTCs are typically present in extremely small numbers in the peripheral blood of cancer patients and their concentration has been shown to be prognostic of subsequent cancer metastasis. ${ }^{2-4}$ Currently, only the CellSearch method, ${ }^{5}$ which depends on EpCAM cell surface markers, has been approved for clinical use by the FDA for CTC isolation. However, because EpCAM surface markers are not present in all tumors and may be lost when cells undergo the epithelial to mesenchymal transition, ${ }^{6}$ the CellSearch method is not universally applicable. Dielectrophoresis (DEP), ${ }^{7}$ a cell surface antigen-independent phenomenon that depends on cell dielectric properties that reflect cell morphology and membrane surface area, ${ }^{8}$ has been widely applied for the characterization of cell dielectric properties and for cell manipulation. ${ }^{9,10}$ Early studies showed that unmodified, viable tumor cells could be separated from

\footnotetext{
a) Author to whom correspondence should be addressed. Electronic mail: pgascoyn@mdanderson.org. Tel.: +1 (713) 8346142. Fax: +1 (713) 834-6103.
} 
erythrocytes and peripheral blood mononuclear cells (PBMNs) by DEP, ${ }^{11-13}$ which has also been shown to have potential for identifying and isolating prostate tumor initiating cells, ${ }^{14}$ oral cancer cells, ${ }^{15,16}$ melanoma, ${ }^{17}$ and colorectal cancer cells. ${ }^{18}$ Most recently, we showed that DEP is able to discriminate between all of the tumor cell types of the NCI-60 panel and PBMNs. ${ }^{19}$ Together, these data suggest that DEP should have wide applicability to isolating CTCs from blood, regardless of their tissue of origin.

In early experiments, differential trapping of tumor versus normal cells was achieved on small microelectrode arrays; ${ }^{11,13}$ however this approach had limited cell discrimination and low throughput capacity, and dielectrophoretic field-flow-fractionation (DEP-FFF) ${ }^{20-23}$ was introduced to allow larger batches of cells to be processed and higher discrimination to be attained. Batch-mode DEP-FFF achieved greater than $90 \%$ efficient isolation of cultured breast cancer cells spiked into $\mathrm{PBMNs}^{21}$ and removed breast tumor cells from $\mathrm{CD} 34^{+}$hemopoietic stem cells with high efficiency. ${ }^{24}$

In batch mode DEP-FFF, an aliquot of cells is allowed to settle to the floor at the inlet end of a separation chamber. Poiseuille flow of eluate is initiated and an AC signal is applied to a microelectrode array covering the chamber floor. Cells reach equilibrium heights under a balance of DEP, sedimentation, and hydrodynamic lift forces and are transported through the separation chamber at rates that reflect the flow velocities at their equilibrium heights. ${ }^{21,25}$ Cells having different dielectric, density, and mechanical properties are thereby eluted at characteristic times and separated in time. In order to achieve good cell discrimination, the size of cell batches processed by a $25 \mathrm{~mm} \times 300 \mathrm{~mm}$ DEP-FFF chamber was found to be limited to around $10^{6}$ cells. $^{21}$ In practice, CTCs are present at concentrations as low as $1 \mathrm{cell} \mathrm{ml}^{-1}$ in the peripheral blood and the minimum specimen volume considered acceptable for CTC analysis is 7 to $10 \mathrm{ml}$. A $10 \mathrm{ml}$ clinical blood specimen contains up to $4 \times 10^{7}$ PBMNs, corresponding to 40 batch-mode DEP-FFF runs, making that technique impractical for routine clinical applications. In this paper, we describe a continuous flow DEP-FFF approach for cell isolation that is capable of processing $4 \times 10^{7}$ PBMNs in less than $1 \mathrm{~h}$, increasing the throughput capacity sufficiently to allow the processing of clinical specimens for CTC analysis.

\section{OVERVIEW OF SEPARATION STRATEGY}

To achieve discrimination between different cell types by DEP, cells must be suspended in a low conductivity medium of physiological osmolarity. This can stress mammalian cells and elicit changes in their dielectric properties as a result of ionic leakage from the cytoplasm if they are suspended in the low conductivity medium for more than $10^{3} \mathrm{~s}^{21}$ This is not a problem in batch-mode DEP-FFF where the cells may be suspended in the eluate before DEP processing begins and processing is completed in around $10^{3} \mathrm{~s}$. In continuous flow DEP-FFF, a large specimen is processed gradually using a continuous injection approach that takes up to an hour. Suspension of the whole specimen in low conductivity medium prior to starting this slow injection process is not feasible if the dielectric properties of the cells are to remain unchanged, and cell discrimination is to remain consistent throughout the run.

To provide for this consistency, we developed the continuous flow DEP-FFF approach shown in Figure 1. Continuous flow of a low conductivity eluate of physiological osmolarity is provided from an inlet port (a) to an outlet port (f), establishing a Poisseuille flow profile in a separation channel of $200 \mathrm{~mm}$ length, $25 \mathrm{~mm}$ width, and $314 \mu \mathrm{m}$ height. In the specimen injection zone (b), the specimen, comprising a cell suspension in physiological medium, is fed through a slot in the bottom of the chamber at a continuous flow rate $q_{i n}$, which is much lower than the eluate inlet flow rate $Q_{i n}$. As a result of the relative flow rates, the specimen forms a thin lamina flowing adjacent to the floor of the chamber beneath the main eluate flow stream. As the thin specimen lamina travels along the chamber floor in the ion-diffusion region, cells find themselves subjected to sedimentation and weak hydrodynamic lift forces and settle to equilibrium heights very close to the chamber floor (see Figure 1(c)). Meanwhile, ions diffuse from the thin specimen lamina into the main eluate flow stream, while non-ionic osmolytes counter-diffuse from the eluate into the specimen lamina. As a result, the high conductivity of 


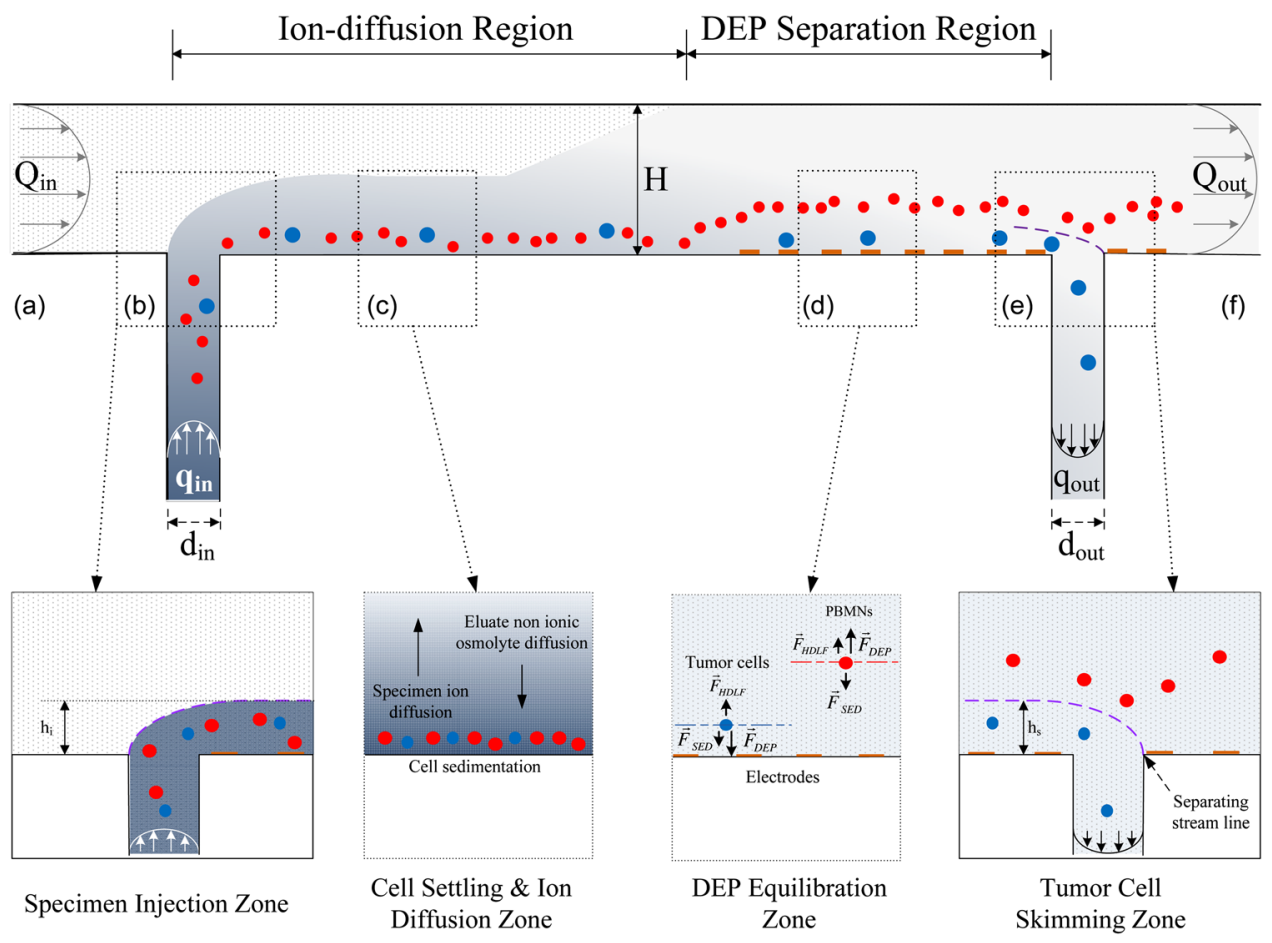

FIG. 1. DEP-FFF isolation of tumor cells from PBMNs in a continuous-flow chamber viewed from the side. The principles of specimen injection, cell settling and specimen deionization, height equilibration by force balance, and isolation of the tumor cells from PBMNs by skimming are described in the text. The relative vertical scale of the extremely thin chamber is exaggerated by $\sim 120$-fold for clarity.

the specimen lamina is reduced, while the osmolarity near the cells is maintained at a physiological level. After flowing a sufficient distance $L_{\text {mix }}$ along the chamber for diffusion to reach equilibrium (see Sec. IV), the ion concentration, and resultant electrical conductivity of the medium, becomes independent of height in the chamber and small enough for DEP separation to be undertaken. The flow then enters the DEP separation region, where microelectrodes on the chamber floor are energized by an appropriate AC voltage to impose DEP forces on the cells. As they flow over the microelectrodes, tumor cells are pulled towards the chamber floor by positive DEP forces, while PBMNs are repelled and levitated by negative DEP forces. Cells eventually move to heights at which the DEP, sedimentation, and hydrodynamic lift forces balance (see Figure 1(d)). Finally, as the flow enters the tumor cell skimming zone (e), fluid is withdrawn through a slot in the chamber floor at a rate $q_{\text {out }}$, skimming off a thin lamina of fluid from the bottom of the chamber up to a height $h_{s}$. Tumor cells, which have reached equilibrium heights close to the chamber floor, are thereby captured through the withdrawal slot while PBMNs, which reached equilibrium heights above the skim height $h_{s}$, are carried over the slot and exit to waste with the main eluate exit flow $Q_{\text {out }}$ at (f). The detailed design and operation of these stages will be described in the following sections.

\section{CONTINUOUS INJECTION AND SKIMMING}

As indicated, the aim in continuous flow DEP-FFF is to transport the specimen along the floor of the separation chamber in a thin lamina that flows beneath the main eluate stream. For the parabolic Poiseuille flow velocity profile, the thickness of this specimen lamina, $h_{i n}$, above the chamber floor is related to the injection flow rate, $q_{i n}$, and the eluate inlet rate $Q_{i n}$ according to the expression $Q_{i n} /\left(Q_{i n}+q_{i n}\right)=3\left(h_{i n} / H\right)^{2}-2\left(h_{i n} / H\right)^{3}$, where $H$ is the height of the chamber. For a very thin specimen lamina, $q_{i n} \ll Q_{i n}$ and $h_{i n} \approx H\left(q_{i n} / 3 Q_{i n}\right)^{1 / 2}$. Similarly at the withdrawal port, a thin lamina of fluid may be skimmed from the chamber floor up to a height $h_{s}$ by withdrawing fluid at a rate $q_{\text {out }}$, where $h_{s} \approx H\left(q_{\text {out }} / 3 Q_{\text {out }}\right)^{1 / 2}$. 
In practice, $q_{\text {in }}$ and $q_{\text {out }}$ are very small and cell sedimentation can result in imperfect injection and skimming behavior if there are either vortices or positions of low flow rate at the injection or withdrawal slots. In order to optimize the slot design, we simulated flow profiles for various chamber heights, slot widths, and flow rates using COMSOL MULTIPHYSICS software (Stockholm, Sweden) assuming that the fluid was incompressible and had a density of $1036 \mathrm{~kg} \mathrm{~m}^{-3}$ and a dynamic viscosity of $1.31 \times 10^{-3} \mathrm{~Pa} \mathrm{~s}$, reflecting the properties of the eluate medium used for our cell isolation experiments. The results for the withdrawal slot simulations are shown in Figure 2. The behavior at the inlet slot region is a mirror image of that at the withdrawal slot.

Desirable skimming behavior was observed when the slot width $d_{\text {out }}$ was small compared with the chamber height $H$ (Figure 2(a)). In this case, a well defined separation region at the slot opening cleanly split the thin lamina of height $h_{s}$ at the chamber bottom from the main chamber flow. Streamlines above the skim height $h_{s}$ travelled essentially horizontally across the slot, and no regions of low flow rate or significant vortices were generated in the separation region. In this case, tumor cells close to the chamber floor would be skimmed cleanly into the withdrawal slot, while PBMNs in the fluid above the skim height would be rapidly carried over the slot without being thrown into the slot by inertial forces or having the opportunity to sediment into the slot from regions of low flow rate.

As the withdrawal slot width $d_{\text {out }}$ was increased, the separation behavior became less ideal (Figure 2(b)). Although the skim height $h_{s}$ still followed the Poiseuille model (equations above), the flow in the slot opening became more complex. Streamlines above the skim height $h_{s}$ travelled downwards into the mouth of the slot causing a depression by a distance $d_{s}$ of the region where the withdrawal and main chamber flow streams split. Furthermore, a vortex of diameter $W_{v}$ formed in the withdrawal tube and significant regions of low flow rate were generated in the separation region. In this case, tumor cells close to the chamber floor would be skimmed

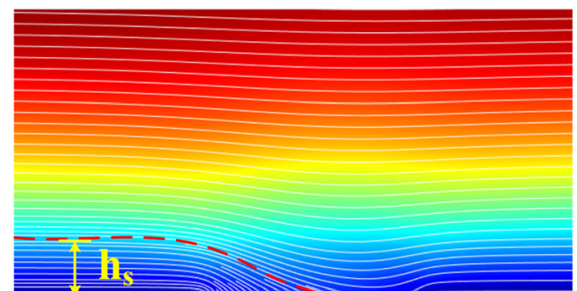

(a) $H=314 \mu \mathrm{m}$,
$d_{\text {out }}=127 \mu \mathrm{m}$,
$Q_{\text {in }}=1000 \mu \mathrm{L} \cdot \mathrm{min}^{-1}$,
$q_{\text {out }}=20 \mu \mathrm{L} \cdot \mathrm{min}^{-1}$

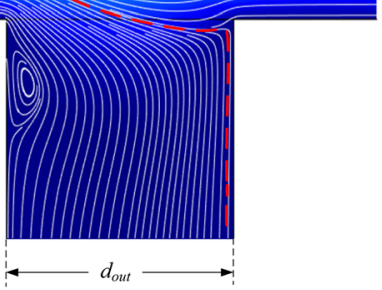

(b)
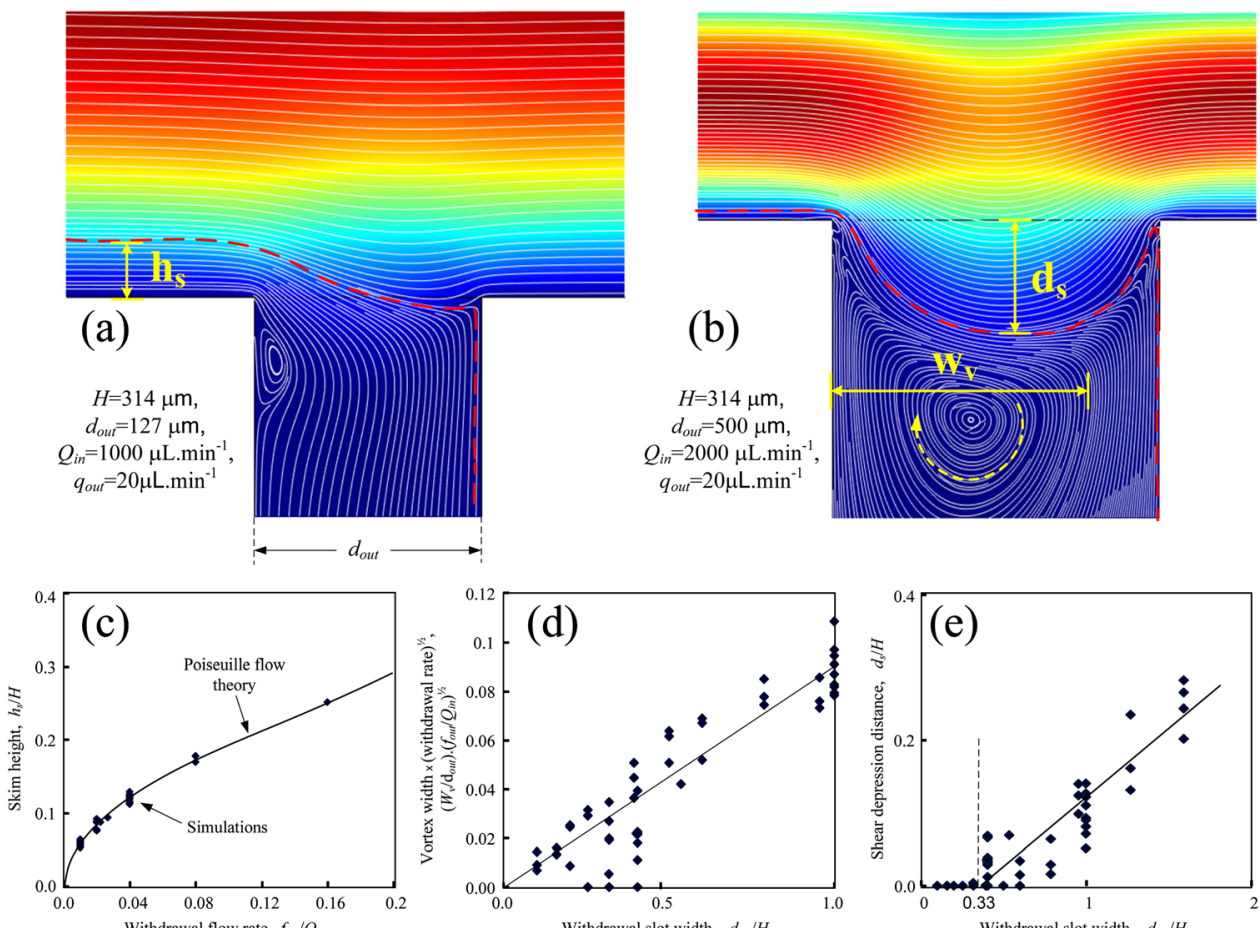

Withdrawal flow rate, $f_{\text {oww }} / O_{\text {in }}$
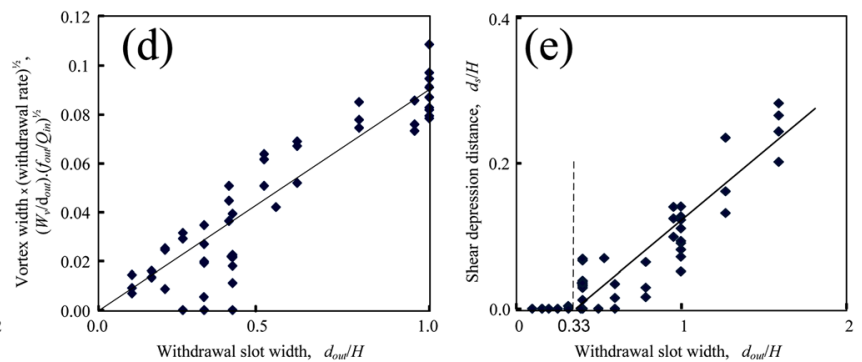

FIG. 2. COMSOL MULTIPHYSICS simulations of the fluid flow behavior at the DEP-FFF chamber withdrawal slot for different geometries and flow rates. (a) When the withdrawal slot width $d_{\text {out }}$ is small compared with the chamber height $H$, optimum skimming behavior is observed with negligible vortices or regions of low flow rate; (b) as the relative withdrawal slot width is increased, a vortex forms within the slot, streamlines from the main channel are depressed into the slot region and zones of low flow rate appear; (c) the skimming height $h_{s}$ accurately follows that predicted by simple Poiseuille flow theory (see text). Vorticity (d) and depression of the streamlines from the main channel (e) increase with increasing slot width. 
into the withdrawal slot but then potentially recirculated in the vortex. Because of the curvature of the streamlines, some may be thrown by inertial forces into the depressed streamlines of the main chamber flow, carried back into the main chamber, and lost to waste. Meanwhile, PBMNs traveling above the skim height that followed the depressed streamlines from the main channel into the chamber mouth would not only have a tendency to be thrown into the withdrawal flow by the curvature of the streamlines but also would need to be carried uphill from their depressed locations well down inside the mouth of the withdrawal slot back into the main chamber against the sedimentation forces acting on them. As a result of these effects, some PBMNs would enter the withdrawal stream and contaminate the tumor cell fraction.

Our simulations establish criteria for designing efficient withdrawal slots and show, in particular, that a withdrawal slot of width $d_{\text {out }}<H / 3$, regardless of the flow rate, exhibits negligible depression of the main chamber streamlines into the mouth of the withdrawal port (Figure 2(e)) as well as low vorticity (Figure 2(d)). Similar principles apply to the injection slot design to prevent the potential for cell accumulation in the mouth of the injection slot. In our experiments, a chamber height of $314 \mu \mathrm{m}$ and injection and withdrawal slot widths of $127 \mu \mathrm{m}$ were chosen, corresponding to the flow conditions shown in Figure 2(a). At the inlet, the specimen was injected at a rate of $q_{\text {in }}=25 \mu \lambda \mathrm{min}^{-1}$ with a main eluate flow rate $\mathrm{Q}_{\text {in }}=1000 \mu \mathrm{lmin}^{-1}$, leading to a specimen lamina thickness $h_{i n}=29.3 \mu \mathrm{m}$ at the inlet. At the outlet, the withdrawal rate was $q_{\text {out }}=20 \mu \lambda \mathrm{min}^{-1}$ leading to a skimming height of $h_{s}=26.4 \mu \mathrm{m}$. In our experiments, the injection and withdrawal flows were provided by $1 \mathrm{ml}$ syringes driven by digital syringe pumps (KDS210, KD Scientific, Holliston, Massachusetts) and the eluate flow by a gear pump (Ismatec, Glattbrugg, Switzerland).

The concept of combining and splitting lamina flow streams in a microfluidic channel is the basis of the so-called $\mathrm{H}$ filter design in which two inlet ports enter from opposite sides at one end of the chamber to combine lamina flows and two withdrawal ports exit from opposite sides at the other end of the chamber to split lamina flows. In cell separation applications, sedimentation of cells is a significant problem at low flow rates and the channels and tubing that carry the cells into and out of the separation chamber should be oriented vertically to avoid cell loss resulting from settling and adherence to tubing surfaces. In the H-filter design ${ }^{26}{ }^{2}$ regions of stagnant flow can occur at both ends of the chamber at the interfaces of the flow streams. At the low flow velocities required for isolation of tumor cells, sedimentation of PBMNs would occur in the slow-moving region of fluid heading towards the top withdrawal port in the $\mathrm{H}$-filter configuration, and some PBMNs would inevitably fall into the lower withdrawal port and contaminate the tumor cell isolate. In the $\pi$ configuration of our continuous flow DEP-FFF chamber described here, the possibility of contamination is greatly reduced because stagnant flow is eliminated from the fluid interface regions. Tumor cells flow directly downward through the withdrawal slot in the chamber floor while PBMNs travel horizontally. Furthermore, microelectrodes are positioned on the chamber floor on both the upstream the downstream sides of the withdrawal slot in our design. These continue to levitate PBMNs by DEP forces in the slot region and on the far side of the slot. This helps reduce still further the likelihood PBMNs will fall into the withdrawal slot and contaminate the tumor cell isolate. In these ways, our $\pi$ configuration improves significantly upon the H-filter design concept for applications involving sedimentary particles such as cells.

\section{DEIONIZATION}

In order to pull tumor cells towards the microelectrodes on the floor of our separation chamber by positive DEP while simultaneously repelling PBMNs high into the eluate flow stream by negative DEP, as required in our cell isolation strategy, the cell suspending medium conductivity must be much lower than the cell cytoplasmic conductivity. ${ }^{9}$ The starting conductivity of our specimens was $1.4 \mathrm{~S} \mathrm{~m}^{-1}$, approximately the same as that of the cell cytoplasms, and this needed to be lowered to a target value of about $60 \mathrm{mS} \mathrm{m}^{-1}$ before the cells could be subjected to DEP separation. The reduction in conductivity was accomplished by using diffusion to deplete the ions in the specimen as it moved through the cell settling and ion diffusion zone shown in Figure 1(c). 
The length of the ion diffusion zone was chosen in accordance with the chamber height $H$ and flow rate $Q_{i n}$ to insure that ions had sufficient time to diffuse throughout the chamber height before the sample entered the DEP zone. The required mixing length $L_{m i x}$ for a diffusible species has been analyzed for the $\mathrm{H}$-filter microfluidic configuration ${ }^{27}$ and, by analogy for our $\pi$ configuration device, may be written as $L_{m i x} \approx H\left(Q_{i n}+q_{i n}\right) /(W D)$, where $W$ is the width of the chamber in the direction perpendicular to the plane of Figure 1 and $D$ is the diffusion coefficient of the diffusing species.

Our cell specimens contained between $20 \times 10^{6}$ and $40 \times 10^{6}$ PBMNs (containing trace levels of tumor cells) collected from whole blood by centrifugation over Histopaque 1077 (Cat 10771-100 ml, Sigma-Aldrich, St Louis, USA) and were suspended in $1 \mathrm{ml}$ RPMI medium that had been adjusted to a density of $1036 \mathrm{~kg} \mathrm{~m}^{-3}$ by adding iodixanol (Optiprep ${ }^{\mathrm{TM}}$ Density Medium D1556, Sigma-Aldrich, St Louis) to a concentration of $11 \%{ }^{21}$ We used the eluate recipe developed in our earlier DEP-FFF studies ${ }^{21,28}$ composed of an aqueous solution of $9.5 \%$ sucrose (S7903, Sigma-Aldrich, St Louis, MO), $0.1 \mathrm{mg} \mathrm{ml}^{-1}$ dextrose (S73418-1, Fisher, Fair Lawn, NJ), 0.1\% pluronic F68 (P1300, Sigma-Aldrich, St Louis, MO), 0.1\% bovine serum albumin (A7906, Sigma-Aldrich, St Louis, MO), $1 \mathrm{mM}$ phosphate buffer $\mathrm{pH}$ 7.0, $0.1 \mathrm{mM}$ CaAcetate, $0.5 \mathrm{mM}$ MgAcetate, and 100 units $\mathrm{ml}^{-1}$ catalase (C30, Sigma-Aldrich, St Louis, MO). This mixture was adjusted to a conductivity of $30 \mathrm{mS} \mathrm{m}^{-1}$ with KCl. Pluronic F-68 provided mechanical stabilization of cell membranes under flow conditions, ${ }^{29}$ catalase protected cells from reactive oxygen intermediates,$^{30}$ and bovine serum albumin inhibited cell adhesion to tubing and chamber surfaces. The specimen and eluate densities were both $1036 \mathrm{~kg} \mathrm{~m}^{-3}$, as a result of their iodixanol and sucrose contents, respectively, and this parity of densities was chosen to avoid the possibility of convection mixing when the flow streams were combined in the continuous flow chamber. After injection into the chamber, the $\mathrm{Na}^{+}, \mathrm{K}^{+}$, and $\mathrm{Cl}^{-}$ions that dominated the high conductivity of the specimen diffused throughout the eluate. Sucrose counter diffused from the eluate to maintain the osmolarity of the cells at a physiological level so they were not osmotically stressed, which would have impacted cell size and membrane dielectric characteristics. ${ }^{28}$

We simulated the diffusion of ions and sucrose in our continuous flow device using the COMSOL MULTIPHYSICS software and the results for the conductivity distributions in key zones of the chamber are shown in Figure 3. In the specimen injection zone (Figure 3(a)), a very large conductivity gradient exists where the specimen stream $\left(1400 \mathrm{mS} \mathrm{m}^{-1}\right)$ first joins the main eluate flow $\left(30 \mathrm{mS} \mathrm{m}^{-1}\right)$. At a distance $18 \mathrm{~mm}$ downstream from the specimen inlet, in the cell settling and ion diffusion zone (Figure 3(b)), the ion conductivity is clearly spreading upwards through the chamber and by the time the flow reaches the DEP equilibration zone $40 \mathrm{~mm}$ downstream from the specimen inlet and the tumor cell skimming zone $80 \mathrm{~mm}$ downstream from the specimen inlet (Figure 3(c)), ion diffusion is essentially complete and the conductivity is homogeneous throughout the chamber height at about $61.5 \mathrm{mS} \mathrm{m}^{-1}$. Sucrose diffusion is also completed before the specimen reaches the DEP equilibration zone.

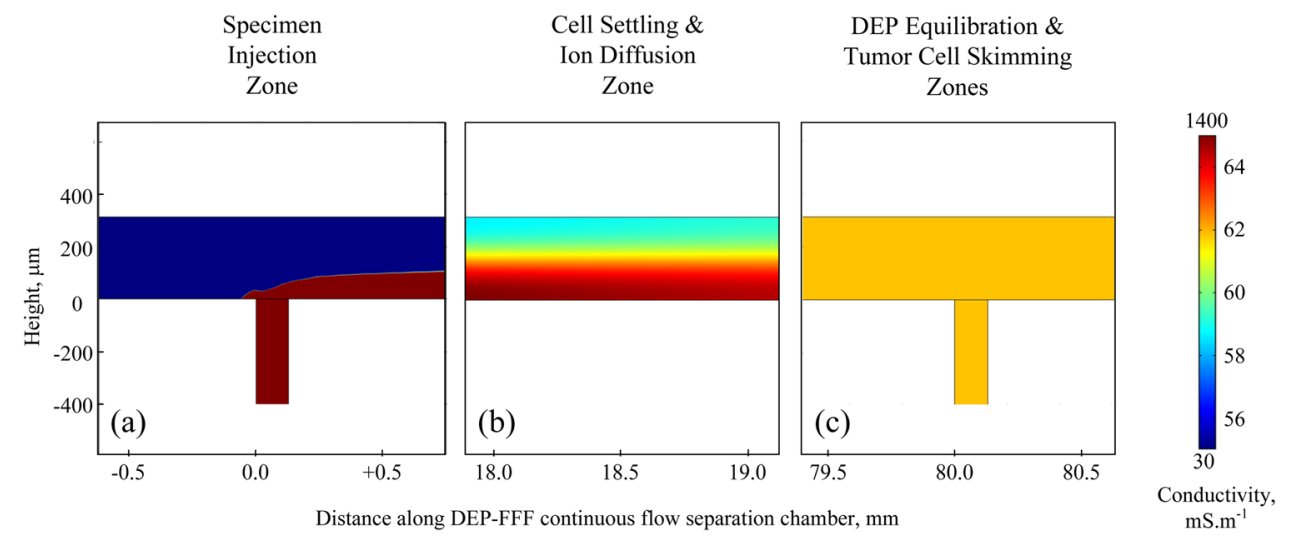

FIG. 3. COMSOL-MULTIPHYSICS simulations of the conductivity distribution in the flow stream (a) at the specimen injection zone, (b) at the midpoint of the cell settling and ion diffusion zone, and (c) at the cell skimming zone. 
To verify these simulation results, we measured the AC current drawn by the microelectrode array. This current depends much more sensitively on the conductivity immediately adjacent to the microelectrode that on the conductivity high in the chamber. We found that the microelectrode current was the same under continuous specimen injection conditions as when the chamber was filled with a homogeneous medium having a conductivity of $61.5 \mathrm{mS} \mathrm{m}^{-1}$. This showed that the ion concentration at the microelectrode plane was the same in both cases, proving that diffusion was complete. Furthermore, we conducted an experiment in which fluid leaving the ion diffusion region was skimmed from the chamber floor up to different heights by altering the withdrawal flow rate. The conductivity of the withdrawn fluid was $61.5 \mathrm{mS} \mathrm{m}^{-1}$ regardless of the skim height, also showing that diffusion was complete.

This method of reducing the conductivity during flow through the ion diffusion zone insured that all cells were subjected to exposure to low conductivity conditions for the same, relatively short, period of time regardless of when they entered the chamber during the $40 \mathrm{~min}$ specimen processing time. Passage of cells through this zone took about $180 \mathrm{~s}$ and this afforded sufficient time for cells to sediment to equilibrium positions close to the chamber floor so that they entered the DEP equilibration zone at identical heights and all were subjected to similar electric field conditions.

\section{DEP STAGE}

The frequency and magnitude of the electrical signal applied to the microelectrodes in the DEP equilibration zone (Figure 1(d)) are chosen so that the tumor cells are pulled towards the chamber floor while the PBMNs are repelled high above it. This is possible because the direction of the DEP force is determined by whether the applied electric field frequency is greater or less than the characteristic "crossover frequency" $f_{c o}$ of a given cell type. ${ }^{10,31,32} \mathrm{In}$ the accompanying article, ${ }^{19}$ we show that the crossover frequencies of all cell types derived from solid tumors in the widely representative NCI-60 panel of cancers lie far below those of all the subpopulations of peripheral blood cells. By choosing a signal frequency that is in between the crossover frequencies of tumor cells and PBMNs, it is possible to impose the desired differential DEP forces that drive cell separation and allow tumor cells to be isolated by skimming them from the chamber floor. At the equilibrium eluate target conductivity of $60 \mathrm{mS} \mathrm{m}^{-1}$ used for our continuous flow DEP-FFF tumor cell isolation experiments, the crossover frequencies $f_{c o}$ of all solid tumor cell types were well below $100 \mathrm{kHz}$ while those of all the blood cell subpopulations were above $200 \mathrm{kHz}$. Therefore, a DEP signal frequency of $130 \mathrm{kHz}$ was chosen for our tumor cell isolation experiments. (Note that the crossover frequency is proportional to the cell medium suspension conductivity, so that the $f_{c o}$ values measured by batch mode DEP-FFF at $30 \mathrm{mS} \mathrm{m}^{-1}$ in the accompanying article ${ }^{19}$ are half the corresponding $f_{c o}$ values at the $60 \mathrm{mS} \mathrm{m}^{-1}$ target conductivity value used for continuous mode isolations in this article.)

The DEP force imposed on cells depends on the square of the applied voltage $V$, suggesting it might be advantageous to use a high DEP voltage to increase the height differential between tumor and PBMNs leaving the DEP equilibration zone. ${ }^{19}$ However, the electric field that gives rise to the DEP force also induces a transmembrane potential difference that can stress cells and cause them to become leaky towards ions and/or electroporated, which could alter their DEP properties and confound their separation characteristics. The magnitude of the induced transmembrane potential difference depends upon the electric field strength, the cell diameter, and the applied electric field frequency. ${ }^{30}$ Because of their larger diameter and closer proximity to the microelectrodes during continuous DEP-FFF isolation, tumor cells are more prone than PBMNs to damage by a high voltage in our DEP-FFF cell isolation experiments. However, we found that we could use an applied voltage of $4 \mathrm{~V}$ p-p in our experiments to provide strong DEP forces without evidence of diminished tumor cell isolation efficiency. Catalase was included in our eluate buffer as in our previous work ${ }^{21,28}$ to protect the cells from a low concentration of reactive oxygen intermediates that may be produced by electrochemical processes at the microelectrodes. ${ }^{30}$ 
To confirm the differential height distributions of tumor cells and PBMNs under force balance conditions, we ran a batch-mode DEP-FFF experiment using the chamber described in earlier work $^{28,33}$ and recorded the time-dependent elution profile of a mixture of PBMNs from healthy donors and MDA-MB-231 human breast cancer cells for a DEP frequency that corresponded to our continuous flow separation conditions. ${ }^{19}$ The cell elution profile was determined using a laser particle counter (PC2400D, ChemTrac Systems, Norcross, Georgia, USA) to count and size the cells as they left the batch-mode chamber. Because the cells were carried through the chamber at the velocities of the eluate at their equilibrium heights, the cell elution times could be mapped directly to the cell equilibrium height distribution in the chamber. The results are shown in Figure 4 and it may be seen that the MDA-MB-231 cells traveled through the DEP-FFF chamber between 5 and $13 \mu \mathrm{m}$ above the chamber floor, while the PBMNs were transported at between 14 and $>40 \mu \mathrm{m}$ height. Tumor cells may be isolated from PBMNs in continuous flow DEP-FFF by using a skim height that is between the tumor cell and PBMNs transport heights under the prevailing DEP field conditions.

In Figure 4, the batch mode DEP-FFF chamber had a microelectrode array area of $7500 \mathrm{~mm}^{2}$ and the power output capacity of our signal generator limited the maximum DEP voltage to $2.8 \mathrm{~V}$ p-p. For continuous flow DEP-FFF, our chamber had a microelectrode array area only one third that size, which permitted us to use the higher operating voltage of $4 \mathrm{~V} \mathrm{p}$-p. The DEP-FFF force balance equation ${ }^{19,28,34}$ showed that the levitation height for PBMNs under those conditions was $>27 \mu \mathrm{m}$. Accordingly, we chose a skimming height of $h_{s}=26 \mu \mathrm{m}$ by setting the withdrawal rate to $q_{\text {out }}=20 \mu \mathrm{l} \mathrm{min}^{-1}$ for continuous flow DEP-FFF cell isolation experiments (as in Figure 2(a)).

In principle, fluid convection cells could form adjacent to energized DEP electrodes as the result of temperature gradients associated with Joule heating of the fluid. Convection cells on our periodic DEP microelectrode array would be expected to have the same spatial periodicity as the DEP force field and it might be possible for convection and DEP fields to act in

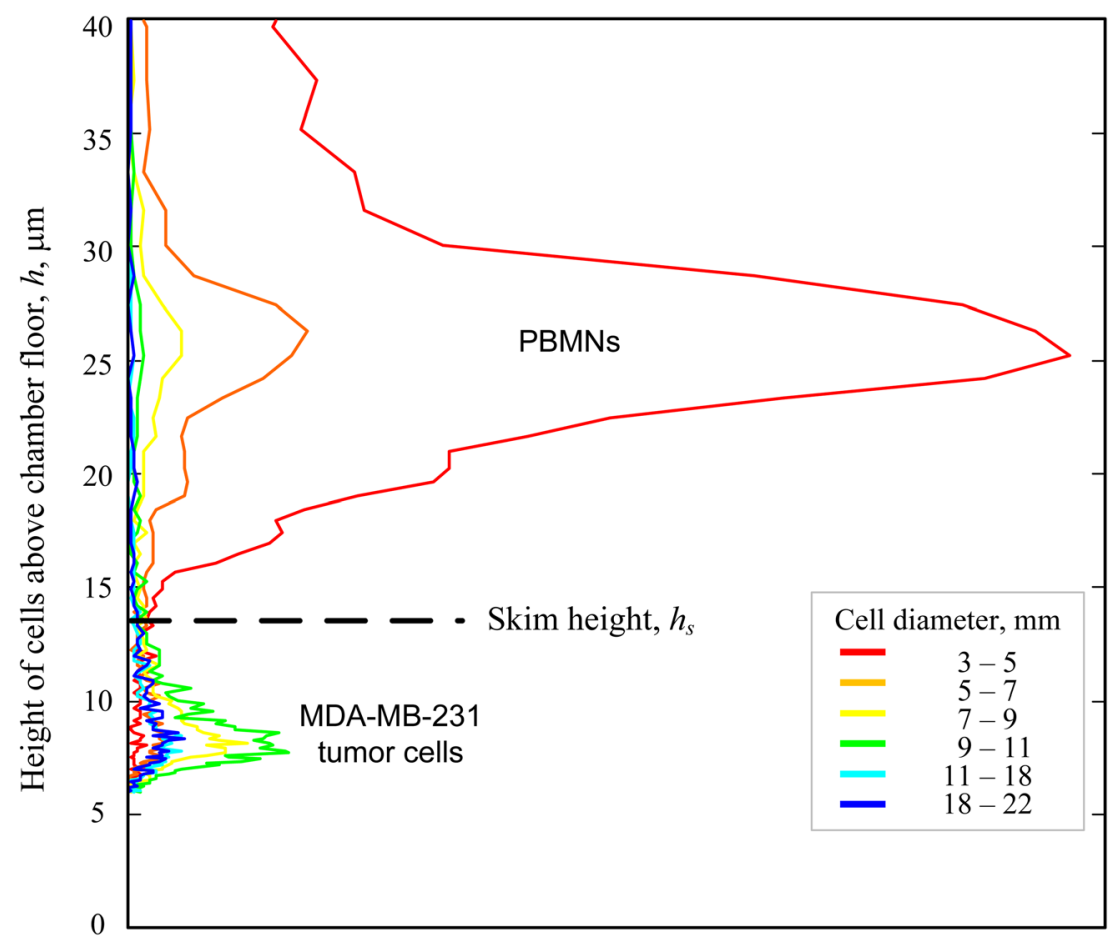

Proportion of cells at given height

FIG. 4. Height distributions of MDA-MB-231 human breast cancer cells and PBMNs as they flowed through the chamber in force-balance equilibrium during batch mode DEP-FFF separation. The height distribution was mapped from the cell elution times assuming that the transit velocities of the cells reflected their heights in the Poiseuille hydrodynamic flow profile inside the DEP-FFF chamber. 
combination to alter the height at which cells are transported over the microelectrode array and thereby impact the cell isolation characteristics. This possibility was neglected in the analytical equations we used to describe DEP-FFF and the effect, if present, seems to be relatively small because one result of it would be to shift the apparent crossover frequency at which the direction of the DEP force on cells appears to reverse in DEP-FFF. In this case, the crossover frequency deduced from DEP-FFF would differ from the true crossover frequency measured by conventional DEP methods. Even though we did not observe such a frequency shift, we believe that this phenomenon is worthy of more careful study in which the modeling work is extended to include coupling of thermal, hydrodynamic and electrokinetic parameters in determining the fluid flow and cell transit behaviors. Future modeling including such coupling could potentiate further improvements to continuous mode DEP-FFF.

\section{TESTS WITH CLINICAL SPECIMENS}

We ran tests of continuous flow DEP-FFF isolation of MDA-MB-435, MDA-MB-231, and other cultured tumor cells spiked into PBMNs from healthy donors to compare with our earlier batch-mode DEP-FFF experiments. ${ }^{21}$ Measurements were conducted using a DEP-FFF chamber $160 \mathrm{~mm}$ long having a width of $25 \mathrm{~mm}$, a height of $314 \mu \mathrm{m}$, and an inlet to outlet slot spacing of $90 \mathrm{~mm}$. The chamber floor was lined by a microelectrode array based on the design detailed earlier $^{33}$ in which parallel gold-on-copper microelectrodes of $50 \mu \mathrm{m}$ width and spacing were patterned on a kaptan substrate. However, only the last $45 \mathrm{~mm}$ of the microelectrode array leading up to the outlet slot was energized. The DEP and flow conditions were as described in earlier sections, namely $2000 \mu \mathrm{lmin}^{-1}$ and $20 \mu \mathrm{min}^{-1}$ for eluate and injection/withdrawal rates, respectively, a DEP signal of $130 \mathrm{kHz}$ at $4 \mathrm{~V} \mathrm{p}-\mathrm{p}$, and a conductivity of the emerging eluate of about $64 \mathrm{mS} \mathrm{m}^{-1}$.

Cultured tumor cells were prelabeled with fluorescent dye (CellTracker Green CMFDA, Life Technologies, Grand Island, New York) and spiked into PBMNs from $10 \mathrm{ml}$ blood specimens at concentrations ranging from 50 to $600 \mathrm{per} \mathrm{ml}$ blood. Using the method, we developed earlier for characterizing batch-mode DEP-FFF separations, ${ }^{24}$ the output port of the DEP-FFF chamber was connected to a modified FACS instrument and the labeled tumor cells were gated and counted by their fluorescence (Ex $488 \mathrm{~nm}, \mathrm{Em} 517 \mathrm{~nm}$ ) as they emerged. These measurements confirmed that, independently of spiking density, tumor cell isolation efficiencies of $70 \%-80 \%$ were achieved. Figure 5 shows scatter plots obtained for MDA-MB-435 cells at three different spiking concentrations. The average collection efficiency was found to be $75 \%$ and independent of spiking density.

Despite these encouraging results using spiked samples, we were made aware that the dielectric properties of the blood of cancer patients might be modified by their disease and treatment regimen. For example, leukocytosis, thrombocytosis, and increased acute phase protein levels in the blood are typically part of a chronic systemic inflammatory response to late stage malignancy. ${ }^{35}$ These factors have the potential to lead to anomalous blood cell subpopulations that
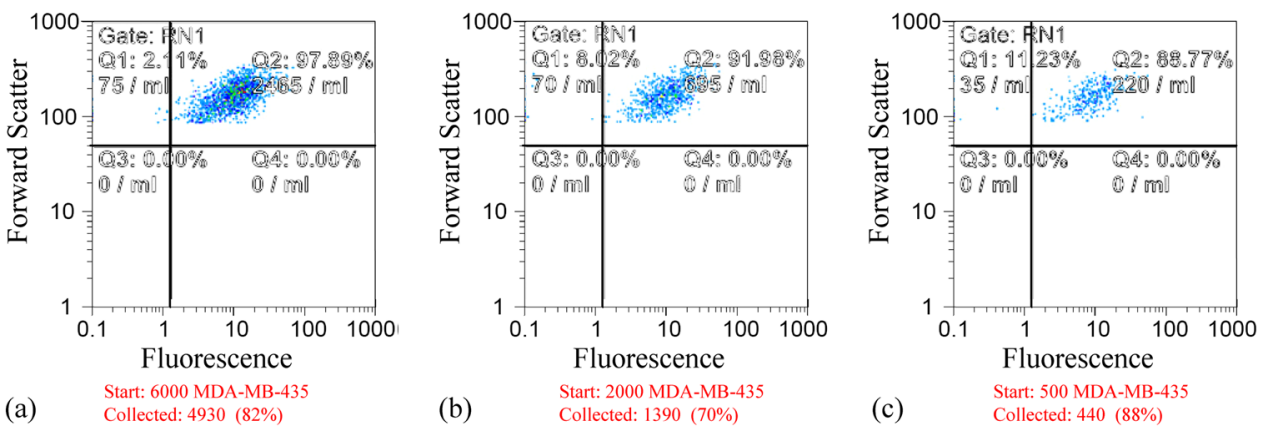

FIG. 5. Flow cytometric (FACS) scattergrams showing the recovery of tumor cells from PBMNs spiked with (a) 6000, (b) 2000, and (c) 500 MDA-MB-435 cultured cells prelabeled with CellTracker Green fluorescent dye. 
might contaminate CTCs during DEP-FFF isolation. Therefore, we conducted a preliminary trial using a limited number of clinical specimens from late stage cancer patients to establish whether CTCs could be isolated from blood under realistic clinical conditions. Peripheral blood specimens were obtained as part of the initiative for molecular profiling in advanced cancer therapy (IMPACT) Trial at The University of Texas M.D. Anderson Cancer Center with informed patient consent and the approval of Institutional Biosafety Committee. Specimens of at least $7 \mathrm{ml}$ volume were collected from patients in $10 \mathrm{ml}$ BD purple cap (EDTA) vacutainers and processed within $3 \mathrm{~h}$. The PBMNs, putatively containing CTCs, were separated from the patient specimens over Histopaque 1077 and subjected to continuous DEP-FFF also using the experimental conditions described in Secs. III-V above. The isolate from the withdrawal slot was collected in a $1 \mathrm{ml} s y-$ ringe for each specimen over the approximately $40 \mathrm{~min}$ processing time.

The FACS approach is not feasible for analyzing CTCs isolated from clinical specimens because a $10 \mathrm{ml}$ blood specimen may contain as few as ten CTCs and very rarely more than hundreds and downstream immunostaining in cell suspension (as opposed to fluorescent dye preloading used to prepare cells before spiking experiments) followed by subsequent FACS analysis are too lossy to use to count such small cell populations accurately. Therefore, a Cytopro $^{\mathrm{TM}}$ instrument (Wescor Model 7620, Logan, Utah) was used to mount the cells from this isolate onto two microscope slides. One slide was immunostained for cytokeratin using FITC-conjugated CK3-6H5 antibodies (Miltenyi Biotec, Bergisch Gladbach, Germany) combined with nuclear staining by DAPI (D1306 Molecular Probes, Eugene, Oregon). An example is shown in Figure 5, where putative CTCs show green fluorescence due to the presence of cytokeratin and both CTC and PBMN nuclei exhibit blue fluorescence.

The primary tumors of all patients enrolled in the IMPACT trial are subjected to screening for somatic mutations associated with their primary cancers. In order to verify the presence of CTCs in our isolates, the unstained slide was subjected to molecular analysis. DNA was extracted from the slide using PicoPure (cat 11815-00, Applied Biosystems) then further cleaned with a QIAamp DNA Micro Kit (cat 56304, Qiagen). The DNA was preamplified using the following primers: Forward: ATGACTGAATATAAACTTGTGGTAGTTGGA, Reverse: GAATTAGCTGTATCGTCAAGGCACT, Vic Reporter: CTTGCCTACGCCACCAG, FAM Reporter: CTTGCCTACGTCACCAG. The Taqman Pre-Amp Master Mix (Cat 4391128) was employed according to the protocol specified by Fluidigm. The sample from the slide, together with a positive and a negative control were tested using a Fluidigm 48.48 Genotyping Array (cat BMK-M-48.48GT, Fluidigm) according to Fluidigm's protocol. For the colon cancer specimen shown in Figure 6, the somatic mutation in the primary tumor was KRAS G13D. MDA-MB-231 was used as a positive control because it possesses this mutation. The specimen exhibited a positive result with a signal intensity that indicated approximately $10 \%$ of the cells on the slide had the KRAS G13D mutation, mirroring the proportion of cells that stained positively for cytokeratin in Figure 6. This not only verified that sufficient CTCs were present on the slide to identify the cancer-causing gene but also shows that the continuous flow DEP-FFF method coupled with molecular analysis may be suitable for clinical screening purposes. A significant advantage of this DEP-FFF CTC isolation approach is that it is label-free and that the isolated CTCs are viable and unmodified. Therefore, these cells are also suitable for a wide range of alternative molecular investigational techniques including gene expression analysis.

\section{DISCUSSION}

Dielectrophoresis is capable of discriminating between blood and tumor cells on the basis of cell size and membrane morphology that contribute to differences in cell membrane area and consequently to cell dielectric properties. ${ }^{8}$ This separation criterion is independent of cell surface protein markers and suggests that DEP provides an approach to isolating CTCs that is applicable to a broad range of cancers. In the accompanying article, we confirm this wide potential applicability by showing that the dielectric properties of all solid tumor cell types in the NCI 60 panel of cancer cell lines, regardless of their tissue of origin, exhibit dielectric properties that are greatly different from those of PBMNs and should allow their isolation from 


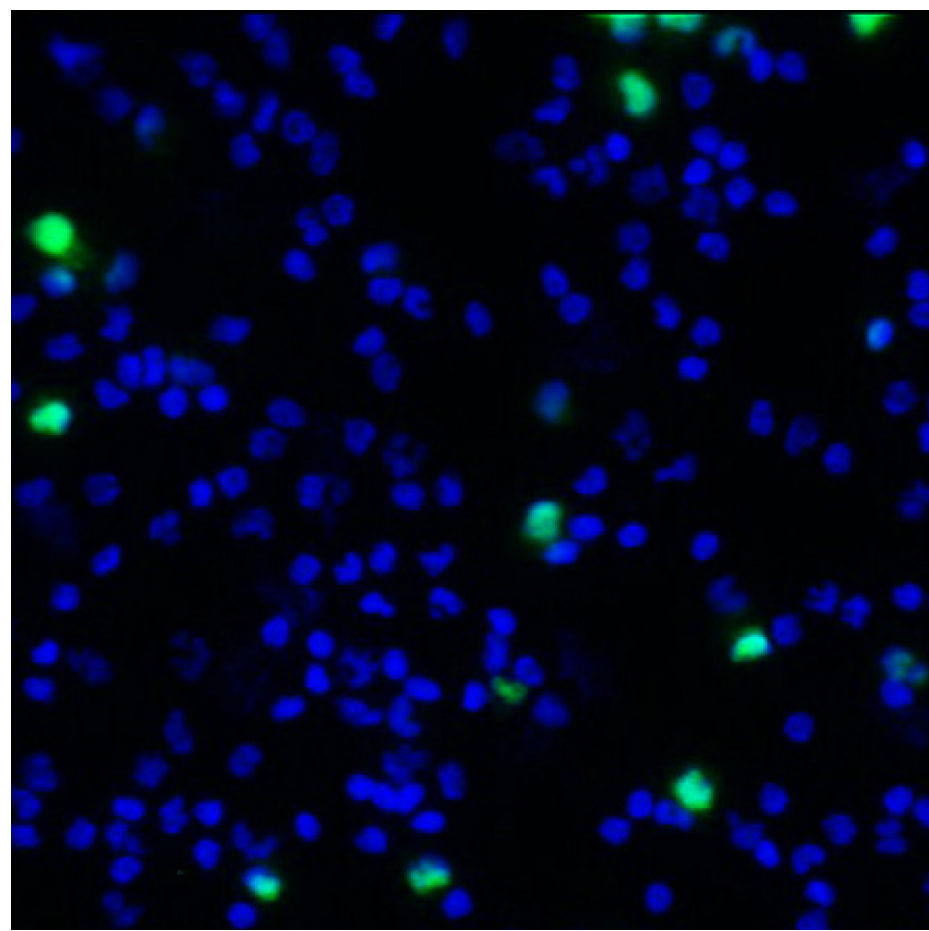

FIG. 6. Circulating tumor cells collected by continuous flow DEP-FFF from the peripheral blood of a patient with colon cancer. The green fluorescence reveals staining of cytokeratin in the tumor cells by FITC-conjugated CK3-6H5 antibodies. PBMNs show only blue fluorescence due to DAPI staining of their nuclei.

blood. ${ }^{19}$ We showed previously that tumor cells may be isolated from the PBMNs of normal donors by batch mode DEP-FFF but that method was capable of handling $<5 \%$ of the desired specimen size for clinical analysis of CTCs. ${ }^{21}$ Here, for the first time, we have detailed all the operational stages of a continuous flow DEP-FFF system that is capable of processing $10 \mathrm{ml}$ clinical specimens in less than $1 \mathrm{~h}$. We have confirmed that this method is capable of not only efficiently isolating cultured cells from PBMNs of healthy donors but also CTCs from clinical specimens derived from cancer patients. Some of the underlying technology presented here has been licensed to ApoCell Inc, which is developing a commercial instrument based on our design concepts. ${ }^{36}$

Our results indicate that continuous flow DEP-FFF may have important applications for clinical CTC analysis. Additional studies need to be undertaken to demonstrate that this new method is as widely applicable as our data suggest and to compare it to competing approaches.

\section{ACKNOWLEDGMENTS}

We are grateful to Ann T. James and Debra L Andrews, phlebotomists in the Department of Investigational Cancer Therapeutics, for her help in coordinating and collecting the clinical specimens. Parts of this work were supported by grant RP100934 from the Cancer Prevention and Research Institute of Texas (CPRIT), by the Kleberg Center for Molecular Markers, and by a sponsored research agreement from ApoCell, Inc. K.S.H. was also supported by a Stand Up to Cancer Dream Team Translational Research Grant, a Program of the Entertainment Industry Foundation (SU2C-AACR-DT0209). P.G. has acted in a consulting capacity to ApoCell, Inc., which has licensed parts of the technology decribed in this work.

${ }^{1}$ M. Cristofanilli, Breast 18(Suppl 3), S38-S40 (2009).

${ }^{2}$ D. C. Danila, M. Fleisher, and H. I. Scher, Clin. Cancer Res. 17, 3903-3912 (2011).

${ }^{3}$ N. J. Meropol, Clin. Adv. Hematol. Oncol. 7, 247-248 (2009).

${ }^{4}$ S. Riethdorf and K. Pantel, Pathobiology 75, 140-148 (2008).

${ }^{5}$ M. C. Miller, G. V. Doyle, and L. W. Terstappen, J. Oncol. 2010, 617421 (2010). 
${ }^{6}$ A. Bonnomet, L. Syne, A. Brysse, E. Feyereisen, E. W. Thompson, A. Noel, J. M. Foidart, P. Birembaut, M. Polette, and C. Gilles, Oncogene 31, 3741-3753 (2012).

${ }^{7}$ H. A. Pohl, Dielectrophoresis: The Behavior of Neutral Matter in Nonuniform Electric Fields (Cambridge University Press, Cambridge, 1978).

${ }^{8}$ X. B. Wang, Y. Huang, P. R. C. Gascoyne, F. F. Becker, R. Holzel, and R. Pethig, Biochim. Biophys. Acta 1193, 330344 (1994).

${ }^{9}$ P. R. C. Gascoyne and J. Vykoukal, Electrophoresis 23, 1973-1983 (2002).

${ }^{10}$ R. Pethig, Biomicrofluidics 4, 022811 (2010).

${ }^{11}$ F. F. Becker, X. B. Wang, Y. Huang, R. Pethig, J. Vykoukal, and P. R. Gascoyne, Proc. Natl. Acad. Sci. U.S.A. 92, 860-864 (1995).

${ }^{12}$ P. R. Gascoyne, X. B. Wang, Y. Huang, and F. F. Becker, IEEE Trans. Ind. Appl. 33, 670-678 (1997).

${ }^{13}$ F. F. Becker, H. Huang, R. Pethig, J. Vykoukal, and P. R. C. Gascoyne, J. Phys. D: Appl. Phys. 27, 2659 (1994).

${ }^{14}$ A. Salmanzadeh, L. Romero, H. Shafiee, R. C. Gallo-Villanueva, M. A. Stremler, S. D. Cramer, and R. V. Davalos, Lab Chip 12, 182-189 (2012).

${ }^{15}$ L. M. Broche, N. Bhadal, M. P. Lewis, S. Porter, M. P. Hughes, and F. H. Labeed, Oral Oncol. 43, 199-203 (2007).

${ }^{16}$ H. J. Mulhall, F. H. Labeed, B. Kazmi, D. E. Costea, M. P. Hughes, and M. P. Lewis, Anal. Bioanal. Chem. 401, 2455-2463 (2011).

${ }^{17}$ A. C. Sabuncu, J. A. Liu, S. J. Beebe, and A. Beskok, Biomicrofluidics 4, 021101 (2010).

${ }^{18}$ F. Yang, X. M. Yang, H. Jiang, P. Bulkhaults, P. Wood, W. Hrushesky, and G. R. Wang, Biomicrofluidics 4, 013204 (2010).

${ }^{19}$ S. Shim, K. Stemke-Hale, J. Noshari, F. Becker, and P. R. C. Gascoyne, Biomicrofluidics 7, 011808 (2013).

${ }^{20}$ X. B. Wang, J. Vykoukal, F. F. Becker, and P. R. Gascoyne, Biophys. J. 74, 2689-2701 (1998).

${ }^{21}$ P. R. C. Gascoyne, J. Noshari, T. J. Anderson, and F. F. Becker, Electrophoresis 30, 1388-1398 (2009).

${ }^{22}$ Y. Huang, X. B. Wang, F. F. Becker, and P. R. Gascoyne, Biophys. J. 73, 1118-1129 (1997).

${ }^{23}$ G. H. Markx, R. Pethig, and J. Rousselet, J. Phys. D: Appl. Phys. 30, 2470-2477 (1997).

${ }^{24}$ Y. Huang, J. Yang, X. B. Wang, F. F. Becker, and P. R. Gascoyne, J. Hematother. Stem Cell Res. 8, 481-490 (1999).

${ }^{25}$ P. R. Gascoyne, Anal. Chem. 81, 8878-8885 (2009).

${ }^{26}$ J. P. Brody, P. Yager, R. E. Goldstein, and R. H. Austin, Biophys. J. 71, 3430-3441 (1996).

${ }^{27}$ H. Bruus, Theoretical Microfluidics (Oxford University Press, Oxford, 2008).

${ }^{28}$ S. Shim, P. Gascoyne, J. Noshari, and K. S. Hale, Integr. Biol. 3, 850-862 (2011).

${ }^{29}$ J. Wu, A. J. Daugulis, P. Faulkner, and M. F. Goosen, Biotechnol. Prog. 11, 127-132 (1995).

${ }^{30}$ X. Wang, J. Yang, and P. R. Gascoyne, Biochim. Biophys. Acta 1426, 53-68 (1999).

${ }^{31}$ T. B. Jones and G. A. Kallio, J. Electrost. 6, 18 (1979).

${ }^{32}$ K. L. Chan, P. R. C. Gascoyne, F. F. Becker, and R. Pethig, Biochim. Biophys. Acta 1349, 182-196 (1997).

${ }^{33}$ J. Vykoukal, D. M. Vykoukal, S. Freyberg, E. U. Alt, and P. R. C. Gascoyne, Lab Chip 8, 1386-1393 (2008).

${ }^{34}$ P. R. C. Gascoyne, "Isolation and characterization of cells by dielectrophoretic field-flow fractionation," in Field-Flow Fractionation in Biopolymer Analysis (Springer-Verlag, 2012).

${ }^{35}$ M. G. Alexandrakis, F. H. Passam, K. Perisinakis, E. Ganotakis, G. Margantinis, D. S. Kyriakou, and D. Bouros, Respir. Med. 96, 553-558 (2002).

${ }^{36}$ V. Gupta, I. Jafferji, M. Garza, V. Melnikova, D. Hasegawa, R. Pethig, and D. Davis, Biomicrofluidics 6, 024133 (2012). 